\title{
Serotonin-Related $F E V$ Gene Variant in the Sudden Infant Death Syndrome Is a Common Polymorphism in the African-American Population
}

\author{
KEVIN G. BROADBELT, MELISSA A. BARGER, DAVID S. PATERSON, INGRID A. HOLM, ELISABETH A. HAAS, \\ HENRY F. KROUS, HANNAH C. KINNEY, KYRIACOS MARKIANOS, AND ALAN H. BEGGS
}

\begin{abstract}
Department of Pathology [K.G.B., D.S.P., H.C.K.], Program in Genomics [M.A.B., I.A.H., K.M., A.H.B.], and The Manton Center for Orphan Disease Research [I.A.H., A.H.B.], Children's Hospital Boston and Harvard Medical School, Boston, Massachusetts 02115; Rady Children's Hospital and Health Center [E.A.H., H.F.K.], University of California, San Diego School of Medicine, San Diego, California 92123
\end{abstract}

\begin{abstract}
An important subset of the sudden infant death syndrome (SIDS) is associated with multiple serotonergic (5-HT) abnormalities in regions of the medulla oblongata. The mouse ortholog of the fifth Ewing variant gene (FEV) is critical for 5-HT neuronal development. A putatively rare intronic variant [IVS2-191_190insA, here referred to as c.128-(191_192)dupA] has been reported as a SIDS-associated mutation in an African-American population. We tested this association in an independent dataset: 137 autopsied cases (78 SIDS, 59 controls) and an additional 296 control DNA samples from Coriell Cell Repositories. In addition to the c.128-(191_192)dupA variant, we observed an associated single-base deletion [c.128-(301306)delG] in a subset of the samples. Neither of the two FEV variants showed significant association with SIDS in either the AfricanAmerican subgroup or the overall cohort. Although we found a significant association of c.128-(191_192)dupA with SIDS when San Diego Hispanic SIDS cases were compared with San Diego Hispanic controls plus Mexican controls $(p=0.04)$, this became nonsignificant after multiple testing correction. Among Coriell controls, 33 of $99(33 \%)$ African-American and 0 of $197(0 \%)$ of the remaining controls carry the polymorphism (c.128-(191_192)dupA). The polymorphism seems to be a common, likely nonpathogenic, variant in the African-American population. (Pediatr Res 66: 631-635, 2009)
\end{abstract}

$\mathrm{T}$ he sudden infant death syndrome (SIDS) is the leading cause of postneonatal infant mortality in the United States today, with an overall incidence of 0.57 of 1000 live births (1). SIDS is defined as the sudden and unexpected death of an infant younger than 12-mo-old that is related to sleep and remains unexplained after a complete autopsy and death scene investigation (2). It is characterized by the death of a seemingly healthy infant during a sleep period, either during sleep itself or during one of the many transitions between sleep and arousal that occurs during a sleep period. Although the cause of SIDS is unknown, it is thought to reflect a combination of environmental and genetic factors that impinge upon an underlying vulnerability (pathophysiological process) in affected

Received May 28, 2009; accepted August 19, 2009

Correspondence: Kevin G. Broadbelt, Ph.D., Department of Pathology, Enders Building Room 1111, Children's Hospital Boston, 300 Longwood Avenue, Boston, MA 02115; e-mail: kevin.broadbelt@ childrens.harvard.edu

Supported by the Deborah Evelyn Barrett Fellowship in SIDS (K.G.B.), CJ Foundation for SIDS (D.S.P.), Scottish Crib Death Foundation (D.S.P.), Christopher James Murphy Foundation, Developmental Disabilities Research Center, Children's Hospital Boston (P30-HD18655), and NIH R01-AR044345 (M.A.B. and A.H.B.). infants to precipitate sudden death $(1,3)$. Indeed, multiple environmental factors are associated with SIDS, including the prone sleep position $(4-6)$, prematurity $(7,8)$, maternal cigarette and alcohol use during pregnancy $(9,10)$, and minor infection (11). However, some exposed infants die of SIDS while others do not, suggesting that, in addition, some infants are genetically at greater risk. Although SIDS is not inherited in a strict Mendelian fashion, the risk for SIDS in subsequent siblings has been estimated to be as high as five times greater than the risk in the general population $(7,12)$. Increased relative risk is certainly consistent with the presence of a strong genetic component, but it is difficult to separate out on a quantitative basis, environmental factors, which undoubtedly also play a major role. Nevertheless, the presence of wellestablished racial and ethnic disparities in SIDS, not completely attributable to socioeconomic factors, suggests that genetic influences play a role in the predisposition to SIDS, especially among the American Indians, African-Americans, New Zealand Maori, and Australian Aborigine $(9,13)$. In addition to predisposing genes, it is possible that rare de novo mutations account for some SIDS cases. Identification of susceptibility and rare causative mutations is of major significance in SIDS research as it could potentially form the basis of genetic screening to identify living infants at risk at birth. Such an advance would allow targeted parental counseling to minimize exposure to environmental risk factors such as prone sleep position or exposure to second hand smoke, i.e., the only currently available types of therapeutic "intervention."

The objective of this study was to evaluate the role of a particular gene, the fifth Ewing variant $(F E V)$ gene, in SIDS. $F E V$ encodes a transcription factor critical for serotonergic (5-HT) neuronal development. The FEV gene is the human homologue of the PET-1 [pheochromocytoma 12 (PC12) E26 transformation specific (Ets)] transcription factor (14-17) identified in rodents and chickens $(18,19)$. In humans, the FEV

\footnotetext{
Abbreviations: 5-HT, 5-hydroxytrptophan (serotonin); ANS, autonomic nervous system; DI, heterozygotes carrying both c.128-(191_192)dupA and c.128-(301_306)delG; $\boldsymbol{F E} \boldsymbol{V}$, fifth Ewing variant gene; I, heterozygotes for the c.128-(191_192)dupA polymorphism; PET-1, [pheochromocytoma 12 (PC12) E26 transformation specific (Ets)] transcription factor; SIDS, Sudden Infant Death Syndrome; wt, common allele homozygotes
} 
gene product has $96 \%$ similarity to the orthologous mouse Pet-1 protein, and it also displays a similar very restricted brain expression pattern in 5-HT neurons $(20,21)$. The Pet-1 ETS factor plays a role in integrating the upstream transcriptional information required for the specification of the 5-HT neuron phenotype (14,17-19,21). Recently, Rand et al. (22) reported an apparently rare intronic mutation (therein designated IVS2-191_190insA) that seemed to be associated with SIDS in African-American cases. This report supported the hypothesis that altered genes that interfere with the development of the brainstem 5-HT system may specifically increase the known higher risk for African-Americans, thereby in part explaining this important racial disparity in SIDS. This is an attractive hypothesis given that we have previously reported developmental 5-HT abnormalities in the medulla oblongata in SIDS cases compared with controls in three independent datasets over the last 20 y (23-26). The serotonin (5hydroxytrptophan: 5-HT) neurotransmitter in the medulla oblongata (lower brainstem) plays a critical role in statedependent homeostatic functions (23-26). We identified multiple abnormalities in SIDS infants in this system, including increased 5-HT cell density and morphologic immaturity, reduced 5-HT1A receptor binding, and reduced 5-HT transporter (5-HTT) binding relative to the number of 5-HT cell bodies (23,25-27). In addition, similar 5-HT receptor deficits in SIDS cases have been confirmed by independent laboratories $(28-30)$.

Given that genes involved in transcriptional regulation of the specification of 5-HT neuronal phenotype are excellent candidates for contributing to the reported biochemical abnormalities of the medullary 5-HT system in SIDS, we considered it critical to confirm the finding of a rare $F E V$ variant by Rand et al. (22) in an independent dataset of SIDS cases and controls. For this reason, we undertook the following study in which we analyzed DNA samples from SIDS and infant control cases autopsied by the San Diego Medical Examiner from 1991 to 2006. To expand the population, we also analyzed additional control DNA samples from the Coriell Cell repositories. In this study, we tested the hypothesis that the FEV variant reported by Rand et al. (22) in African-American SIDS infants is associated with SIDS in the independent San Diego cohort.

\section{METHODS}

Clinical database. Seventy-eight brainstems (33 females, 45 males) were accrued from infants diagnosed with SIDS from 1991 to 2006 in the Office of the Chief Medical Examiner, San Diego, CA. SIDS was defined as reported (2). Fifty-nine control brainstems from 14 females and 45 males younger than
12 mo were used in line with the age limit specified in the definition of SIDS. These controls died of known causes (established at autopsy, e.g., severe infections, accidents). Two hundred and ninety-six (296) DNA samples from living controls were obtained from the racially-defined Human Variation Panels available from the Coriell Institute Cell Repository (Camden, NJ). The samples currently used are from the Human Variation Collection (HD100CAU, HD100AA, and HD100MEX, HD100CHI) in which genotypes of the human variation panels are listed by self-reported ethnicity. The Children's Hospital Boston Institutional Review Board has approved this study. The tissues from infants dying suddenly and unexpectedly in the San Diego Medical Examiner's systems were accrued under the California autopsy law (California Law Chapter 955, Statutes of 1989 [SB 1069]) that allows the use of such tissues for deidentified research without parental permission.

DNA preparation and sequence analysis. Genomic DNA was isolated using a standard Puregene solid tissue protocol (Genomic DNA Purification Kit, Gentra Systems). The region in the FEV gene where the polymorphism was previously reported was amplified by PCR using primer pairs 5'TATCTGTCCTTGCTCGCCTTGGAA-3' (forward) (22) and 5'GGGGGAGGAAAAGTGAAGT-3' (reverse) designed in our laboratory using Primer Express v3.0, the PCR protocol used was previously described by Rand et al. (22). All products were purified using Exo-Sap as recommended by standard manufactures protocol (Cat \# USB 78201); $5 \mu \mathrm{L}$ of purified products were run on $2 \%$ agarose gel for visualization. Purified products were then sequenced in the Children's Hospital Boston, Genomics Program \& Molecular Genetics Core. Both forward and reverse primers were sequenced, and the results were manually reviewed by three individuals to ensure accuracy of variant identification. Samples with and without the polymorphism were randomly selected and repeated to recheck the findings.

Statistical analysis and genetic nomenclature. Allele frequencies and haplotype frequencies were compared using $\chi^{2}$ tests and Fisher's exact test. Statistical significance was set at $\alpha=0.05$. FEV gene variants are reported following current nomenclature conventions (31) as updated at http:// www.hgvs.org/mutnomen/using NM_017521 as the FEV reference sequence and numbering coding regions bases by the convention whereby the " $\mathrm{A}$ " of the initiating ATG (methionine) codon is base +1 . Under this scheme, the previously designated IVS2-191_190insA variant (22) is herein referred to as c.128-(191_192)dupA.

\section{RESULTS}

Clinical database. The dataset from the Office of the San Diego Medical Examiner was racially and ethnically diverse. There were a total of 78 diagnosed SIDS cases comprised of 30 Caucasians (19 males, 11 females), 34 Hispanics (16 males, 18 females), 7 African-Americans (5 males, 2 females), 4 Asian/Pacific Islanders (4 males), and 3 "other" ethnicity (1 male, 2 females) (Table 1). Exclusion criteria for this study were infants in whom the classification of death could not be unequivocally determined upon completion of the autopsy and death scene investigation, i.e., unclassified cases (2). There were 59 autopsy controls in the dataset comprised of 26 Caucasians (19 males, 7 females), 19 Hispanics (13 males, 6 females), 9 African-American (7 males, 2 females), 2 American Indian/Alaskan (2 males), and 3 "other" (2 males, 1 female) (Table 1). The Coriell racially-defined Human Variation Panels were comprised of North American Caucasian

Table 1. Ethnic distribution of FEV genotypes among cases and controls from the San Diego Chief Medical Examiner's cohort

\begin{tabular}{|c|c|c|c|c|c|c|c|c|c|c|c|c|}
\hline \multirow[b]{2}{*}{ Genotypes* } & \multicolumn{2}{|c|}{ Hispanic } & \multicolumn{2}{|c|}{ Caucasian } & \multicolumn{2}{|c|}{$\begin{array}{l}\text { African- } \\
\text { American }\end{array}$} & \multicolumn{2}{|c|}{ Asian } & \multicolumn{2}{|c|}{ Other } & \multicolumn{2}{|c|}{$\begin{array}{c}\text { Native } \\
\text { American }\end{array}$} \\
\hline & SIDS & $\overline{\text { Cont }}$ & SIDS & $\overline{\text { Cont }}$ & SIDS & $\overline{\text { Cont }}$ & SIDS & $\overline{\text { Cont }}$ & SIDS & $\overline{\text { Cont }}$ & SIDS & Cont \\
\hline $\mathrm{wt} / \mathrm{I}$ & 1 & 1 & 0 & 1 & 1 & 1 & 1 & 0 & 0 & 0 & 0 & 0 \\
\hline wt/DI & 2 & 0 & 0 & 0 & 0 & 0 & 0 & 0 & 0 & 0 & 0 & 0 \\
\hline wt/wt & 31 & 18 & 30 & 25 & 6 & 8 & 3 & 0 & 3 & 3 & 0 & 2 \\
\hline
\end{tabular}

* No homozygotes for either variant were identified so numbers represent individuals heterozygous for the indicated variants.

I, c.128-(191_192)dupA; D, c.128-(301_306)delG; wt, wild type. 
(HD100CAU) (51 males, 49 females), African-American (HD100AA) (17 males, 83 females), Mexican-American community of Los Angeles (HD100MEX) (50 males, 50 females), and the Han People of Los Angeles (HD100CHI) (4 males, 3 females). With the exception of one 8-month-old infant, and two children of 5 and $6 \mathrm{y}$ age in the Caucasian panel, all these individuals were sampled as living adults, thereby obviously excluding the classification of SIDS for all except the 8-moold infant. Repeating the analysis with and without the 8-moold infant does not change the association results. Eleven cases from the Coriell panels failed to amplify for various technical reasons; four from the Caucasian (HD100CAU) panel, one case from the African-American (HD100AA), and six from the Mexican-American panel HD100MEX.

Genetic findings. We observed the previously reported FEV insertion variant, c.128-(191_192)dupA, a single-base insertion 191 base pairs upstream of the $5^{\prime}$ exon 3 splice site (22), in five SIDS cases and three controls, representing several ethnic groups (Table 1). In contrast to the report by Rand et al. (22), where c.128-(191_192)dupA was observed exclusively among African-American SIDS cases, we found this change was relatively common among African-Americans regardless of diagnosis, i.e., whether or not the classification was SIDS or controls (Table 2). Thirty-two of 99 (32\%) African-American control individuals from the Coriell Human Variation Panel were heterozygous for this change. One hundred ten base pairs $5^{\prime}$ to this, in the same intron, we observed an additional, not previously reported, polymorphism, c.128-(301_306)delG, that was always associated with c.128-(191_192)dupA. In our data set, the deletion polymorphism seems to be in complete linkage disequilibrium with the insertion polymorphism in the entire sample, including both cases and controls (Tables 1 and 2). All individuals ( $n=10,2$ cases; 8 African-American Coriell controls) carrying the deletion were heterozygous for both alleles, insertion c.128-(191_192)dupA and deletion c.128-(301_306)delG. Thus, the deletion seems to occur exclusively on chromosomes carrying the c.128-(191_192)dupA insertion. We never observed either allele in the homozygous state. Thus, there were only three genotyping categories: common allele homozygotes "wt," heterozygotes for the c.128-(191_192)dupA polymorphism "I," and heterozygotes carrying both c.128-(191_192)dupA and c.128-(301_306)delG "DI." Under Hardy-Weinberg equilibrium, we expected a small number of homozygotes for the c.128-(191_192)dupA polymorphism; however, given the small sample size of our population, the deficit in homozygotes was not significant

Table 2. Ethnic distribution of FEV genotypes among racially-defined Human Variation Panels from the Coriell Cell Repository

\begin{tabular}{ccccc}
\hline Genotypes* & African-American & Caucasian & Asian & Mex \\
\hline wt/I & 24 & 0 & 0 & 0 \\
wt/DI & 8 & 0 & 0 & 0 \\
wt/wt & 67 & 96 & 7 & 94 \\
\hline
\end{tabular}

* No homozygotes for either variant were identified so numbers represent individuals heterozygous for the indicated variants.

Mex, Mexican American community of Los Angeles; I, c.128(191_192)dupA; D, c.128-(301_306)delG; wt, wild type. under an exact Hardy-Weinberg equilibrium test $(p>0.068)$ (32). We supplemented the relatively limited number of controls available through the San Diego Medical Examiner with controls acquired from the Coriell repository. To assess the statistical likelihood of any associations, we present $p$ values for case-control comparisons twice: once using only San Diego controls (54 total, Table 3) and once after combining San Diego and Coriell controls (350 total, Table 4). We tested within ethnic groups only if there was at least one case of SIDS in the database: Caucasian, African-American, Hispanic/Mexican, and Asian. In total, we performed two haplotype tests and one allele test (Tables 3 and 4). Under the assumption of complete linkage disequilibrium, and hence coupling between the two variants, we tested the presence of the two haplotypes, wt/I or wt/DI against all other haplotypes. In addition, we performed an allele test for I alone, i.e., comparing chromosomes that carry the insertion against all other chromosomes. The allele test for the deletion alone was redundant in this dataset because it corresponded exactly to the DI haplotype test. None of the tests comparing allele or haplotype frequencies in SIDS cases to San Diego controls gave significant $p$ values (Table 3 ). Similar results were obtained when the control group was expanded to include the racially-defined Coriell Human Variation Panels (Table 4) with the exception that the frequency of c.128-(191_192)dupA in San Diego Hispanic SIDS cases was statistically greater than in San Diego Hispanic plus Los Angeles MexicanAmerican controls $(p=0.04)$.

\section{DISCUSSION}

Neurochemical studies indicate an association between SIDS and medullary 5-HT abnormalities in $\sim 70 \%$ of cases, mandating the elucidation of potential abnormalities in the genetic program of 5-HT neuronal development in this disorder $(23,25,26)$. For this reason, we found it imperative to replicate the important study of Rand et al. (22) in which a rare insertion variant in the $F E V$ gene was reported exclusively in SIDS cases of African-American descent. AfricanAmerican infants are more than twice as likely than nonHispanic white infants to die of SIDS (33); the SIDS rate for African-American infants is 1.15 of 1000 compared with 0.49 of 1000 in U.S Caucasian populations (1). The documentation of a SIDS-related gene in African-Americans, e.g., the FEV insertion polymorphism, would be a major step toward ex-

Table 3. p values using only autopsy controls accrued by the Chief Medical Examiner, San Diego

\begin{tabular}{lccccc}
\hline & Hispanic & Caucasian & African-America & Asian & All \\
\hline Haplotype I_wt & 1.000 & 0.464 & 1.000 & 1.000 & 0.697 \\
Haplotype DI & 0.536 & 1.000 & 1.000 & 1.000 & 0.511 \\
Allele I & 1.000 & 0.464 & 1.000 & 1.000 & 1.000 \\
\hline
\end{tabular}

In each case, we test the frequency of a variant, haplotype, or allele, in cases and controls using Fischer's exact test. Of the possible four haplotypes (wt/wt, $\mathrm{I} / \mathrm{wt}, \mathrm{wt} / \mathrm{D}, \mathrm{I} / \mathrm{D})$, the wt/D haplotype was never observed. Thus, we performed only two haplotype tests: I/wt and I/D. In addition, we perform an allele test for the I polymorphism. We compare the frequency of the I allele independent of the presence of the D polymorphism.

I, c.128-(191_192)dupA; D, c.128-(301_306)delG; wt, wild type. 
Table 4. p-values using autopsy controls accrued by the Chief Medical Examiner, San Diego, and Coriell controls

\begin{tabular}{lccccc}
\hline & Hispanic & Caucasian & $\begin{array}{c}\text { African- } \\
\text { American }\end{array}$ & Asian & All \\
\hline Haplotype I_wt & 0.410 & 1.000 & 1.000 & 0.364 & 0.336 \\
Haplotype DI & 0.053 & 1.000 & 1.000 & 1.000 & 0.692 \\
Allele I & 0.040 & 1.000 & 0.699 & 0.364 & 0.524 \\
\hline
\end{tabular}

In each case, we test the frequency of a variant, haplotype, or allele, in cases and controls using Fischer's exact test. Of the possible four haplotypes (wt/wt, I/wt, wt/D, I/D), the wt/D haplotype was never observed. Thus, we performed only two haplotype tests: I/wt and I/D. In addition, we perform an allele test for the I polymorphism. We compare the frequency of the I allele independent of the presence of the D polymorphism.

I, c.128-(191_192)dupA; D, c.128-(301_306)delG; wt, wild type.

plaining at the biologic level, the increased risk for SIDS in African-Americans. However, in contrast to the previous report (22), we observed FEV c.128-(191_192)dupA in both SIDS and controls from all ethnic groups, not just SIDS cases of African-American descent. Approximately one-third of African-American control individuals were heterozygous for c.128-(191_192)dupA demonstrating that this variant is in fact a common polymorphism among African-Americans. Furthermore, we found no evidence for a preferential association of this change with SIDS in the African-American population. We also observed a novel variant c.128-(301_306)delG in eight of the 99 Coriell African-American controls, as well as in two autopsied infant controls. This single-base intronic deletion is in perfect linkage disequilibrium with c.128(191_192)dupA, implying that it arose on an ancestral chromosome carrying the insertion polymorphism. As with the previously identified variant, c.128-(301_306)delG was also not associated with SIDS in African-Americans, but its clinical significance is unknown as we have limited power to investigate the influence of this second polymorphism on SIDS phenotype given the low incidence of the polymorphism in cases and controls and our limited sample size.

It is important to consider possible differences between the current study and that by Rand et al. (22) that may account for our inability to replicate the association of c.128-(191_192)dupA with SIDS in African-Americans. Clearly, these two studies report very different allele frequencies of the insertion polymorphism in the two sets of African-American populations; we identified it in 32 of 99 (32\%) African-American control individuals, whereas Rand et al. found it in only six of $98(6 \%)$ total (zero of 49 nonSIDS controls). The difference between the two sets of controls is too large to be attributed to statistical sampling errors alone $\left(p<1.36 \times 10^{-6}\right.$ comparing the two sets of controls). Possible explanations include technical differences in the assay and/or inaccuracies in base calling, inaccurate or inconsistent designations of racial affinity, and/or difference in the cases and controls used. Rand et al. investigated the entire $F E V$ gene; exons, intron-exon boundaries, and promoter sequence. We sought to replicate only their positive findings; thus, we assayed a single amplicon containing the reported insertion polymorphism. However, except for a slight difference in the reverse primer used in our study compared with Rand et al., the experimental conditions were essentially the same and were therefore un- likely to account for any discrepancy between the findings of the two studies. Both the groups used direct DNA sequencing on both strands of genomic PCR products and we believe the likelihood of extensive miss typing due to technical artifact should be quite low given the robust nature of the technologies used. The origin of African-American cases and controls in the study by Rand et al. (22) is unknown, but, fortuitously, the Coriell Human Variation Panel of self-identified AfricanAmericans is well-characterized genetically. Of 89 specimens reported in detail, the average contribution of African ancestry was $79 \%$ with $21 \%$ Caucasian contribution (34). However, it is notable that the estimated genome-wide proportion of European ancestry among these specimens varied widely, from $1 \%$ to $62 \%$. Thus, studies of admixed populations, such as African-Americans, require large sample sizes to avoid obtaining a nonrepresentative sample. Consequently, one contributing reason for differences between the two studies may have arisen from the possibility that different, nonrepresentative samples of the population were studied by the two groups.

Although the overall sample size (78 SIDS cases and 355 controls) in our study was larger than the report by Rand et al. (96 SIDS cases and 96 controls of which about half were African-American), only seven African-American SIDS cases were available in our database. Thus, our power to detect association within the African-American subset was significantly lower than that of Rand et al. Nevertheless, our observation that roughly one-third of self-identified AfricanAmericans are heterozygous for c.128-(191_192)dupA unequivocally demonstrates that this is a common polymorphism among African Americans and cannot be interpreted as a rare causative mutation. Regarding SIDS phenotype definition, it is possible that the difference in the classification of infant deaths accounted in small part for the different results, given that some cases (dying while awake) were potentially labeled as SIDS by Rand et al. but not in our study. Rand et al. classified cases according to the 1991 NICHD definition (35); in our study, we classified cases according to the 2004 schema of Krous et al. that, unlike the NICHD definition, requires sudden death to be linked to a sleep period.

Of interest, we found a significant association for the insertion polymorphism within the SIDS cases in a Hispanic subset of our database. We caution, however, that the label Hispanic, like "African-American" applies to a heterogeneous group, often of mixed ancestry. Therefore, it is possible that the association signal was due to admixture, in particular with African-American ancestry, among SIDS cases. The Coriell HD100Mex panel is derived exclusively from the Mexican American community of Los Angeles and may contain a smaller African contribution than the broader Hispanic population of SIDS cases and controls collected by the San Diego Medical Examiner's office. Furthermore, the association in our study was significant only before multiple testing correction and no other test showed a significant association for c.128-(191_192)dupA or c.128-(301_306)delG whether testing within groups or the entire sample. Therefore, unless and until this result can be replicated and confirmed in an independent data set, we conclude that there is little evidence to support an 
association of either polymorphism with SIDS in the existing datasets.

In conclusion, we were not able to replicate an association between the previously reported insertion mutation in the FEV gene and SIDS in African-American infants. We caution, however, that our failure to confirm this finding in an independent SIDS dataset does not negate the potential significance of the FEV gene to SIDS, particularly to its relationship with medullary 5-HT abnormalities in affected cases. Further work in SIDS is needed to explore the known functional regions of the $F E V$ gene, as abnormalities here could still lead to aberrant functioning of 5-HT neurons. Also, the interactions between the $F E V$ transcription factor and other transcription factors related to the 5-HT neuronal development cascade, e.g., $\operatorname{lmx} 1 \mathrm{~b}$ (16), GATA2 (15), need to be considered (36). We still believe a full genetic screen of the $F E V$ gene is needed to gain complete insight into the molecular pathogenesis of the potential association between this important gene, SIDS, and medullary 5-HT abnormalities.

Acknowledgments. We thank Mr. Dean R. Campagna for his technical assistance in the amplification of the FEV primer designs and sequencing and evaluations. We are grateful to Dr. Christina Stanley, Deputy Chief Medical Examiner, and the Office of the Chief Medical Examiner, San Diego, CA, for assistance in the accrual of tissue samples.

\section{REFERENCES}

1. Mathews TJ, MacDorman MF 2006 Infant mortality statistics from the 2003 period linked birth/infant death data set. Natl Vital Stat Rep 54:1-29

2. Krous HF, Beckwith JB, Byard RW, Rognum TO, Bajanowski T, Corey T, Cutz E, Hanzlick R, Keens TG, Mitchell EA 2004 Sudden infant death syndrome and unclassified sudden infant deaths: a definitional and diagnostic approach. Pediatrics 114:234-238

3. Filiano JJ, Kinney HC 1994 A perspective on neuropathologic findings in victims of the sudden infant death syndrome: the triple-risk model. Biol Neonate 65:194-197

4. Engelberts AC, de Jonge GA 1990 Choice of sleeping position for infants: possible association with cot death. Arch Dis Child 65:462-467

5. Fleming PJ, Gilbert R, Azaz Y, Berry PJ, Rudd PT, Stewart A, Hall E 1990 Interaction between bedding and sleeping position in the sudden infant death syndrome: a population based case-control study. BMJ 301:85-89

6. Willinger M, Hoffman HJ, Hartford RB 1994 Infant sleep position and risk for sudden infant death syndrome: report of meeting held January 13 and 14, 1994, National Institutes of Health, Bethesda, MD. Pediatrics 93:814-819

7. Hunt CE 2001 Sudden infant death syndrome and other causes of infant mortality: diagnosis, mechanisms, and risk for recurrence in siblings. Am J Respir Crit Care Med 164:346-357

8. Hunt CE, Hauck FR 2006 Sudden infant death syndrome. CMAJ 174:1861-1869

9. Iyasu S, Randall LL, Welty TK, Hsia J, Kinney HC, Mandell F, McClain M, Randall B, Habbe D, Wilson H, Willinger M 2002 Risk factors for sudden infant death syndrome among northern plains Indians. JAMA 288:2717-2723

10. Mitchell EA, Milerad J 2006 Smoking and the sudden infant death syndrome. Rev Environ Health 21:81-103

11. Vege A, Ole Rognum T 2004 Sudden infant death syndrome, infection and inflammatory responses. FEMS Immunol Med Microbiol 42:3-10

12. Oyen N, Skjaerven R, Irgens LM 1996 Population-based recurrence risk of sudden infant death syndrome compared with other infant and fetal deaths. Am J Epidemiol 144:300-305
13. Moon RY, Horne RS, Hauck FR 2007 Sudden infant death syndrome. Lancet 370:1578-1587

14. Cheng L, Chen CL, Luo P, Tan M, Qiu M, Johnson R, Ma Q 2003 Lmx 1b, Pet-1, and Nkx2.2 coordinately specify serotonergic neurotransmitter phenotype. J Neurosci 23:9961-9967

15. Craven SE, Lim KC, Ye W, Engel JD, de Sauvage F, Rosenthal A 2004 Gata2 specifies serotonergic neurons downstream of sonic hedgehog. Development 131:1165-1173

16. Ding YQ, Marklund U, Yuan W, Yin J, Wegman L, Ericson J, Deneris E, Johnson RL, Chen ZF $2003 \mathrm{Lmx} 1 \mathrm{~b}$ is essential for the development of serotonergic neurons. Nat Neurosci 6:933-938

17. Hendricks TJ, Fyodorov DV, Wegman LJ, Lelutiu NB, Pehek EA, Yamamoto B, Silver J, Weeber EJ, Sweatt JD, Deneris ES 2003 Pet-1 ETS gene plays a critical role in 5-HT neuron development and is required for normal anxiety-like and aggressive behavior. Neuron 37:233-247

18. Scott MM, Krueger KC, Deneris ES 2005 A differentially autoregulated Pet-1 enhancer region is a critical target of the transcriptional cascade that governs serotonin neuron development. J Neurosci 25:2628-2636

19. Scott MM, Wylie CJ, Lerch JK, Murphy R, Lobur K, Herlitze S, Jiang W, Conlon RA, Strowbridge BW, Deneris ES 2005 A genetic approach to access serotonin neurons for in vivo and in vitro studies. Proc Natl Acad Sci USA 102:16472-16477

20. Maurer P, Rorive S, de Kerchove d'Exaerde A, Schiffmann SN, Salmon I, de Launoit Y 2004 The Ets transcription factor FEV is specifically expressed in the human central serotonergic neurons. Neurosci Lett 357:215-218

21. Pfaar H, von Holst A, Vogt Weisenhorn DM, Brodski C, Guimera J, Wurst W 2002 mPet-1, a mouse ETS-domain transcription factor, is expressed in central serotonergic neurons. Dev Genes Evol 212:43-46

22. Rand CM, Berry-Kravis EM, Zhou L, Fan W, Weese-Mayer DE 2007 Sudden infan death syndrome: rare mutation in the serotonin system FEV gene. Pediatr Res 62:180-182

23. Kinney HC, Filiano JJ, White WF 2001 Medullary serotonergic network deficiency in the sudden infant death syndrome: review of a 15-year study of a single dataset. J Neuropathol Exp Neurol 60:228-247

24. Kinney HC, Myers MM, Belliveau RA, Randall LL, Trachtenberg FL, Fingers ST, Youngman M, Habbe D, Fifer WP 2005 Subtle autonomic and respiratory dysfunction in sudden infant death syndrome associated with serotonergic brainstem abnormalities: a case report. J Neuropathol Exp Neurol 64:689-694

25. Panigrahy A, Filiano J, Sleeper LA, Mandell F, Valdes-Dapena M, Krous HF, Rava LA, Foley E, White WF, Kinney HC 2000 Decreased serotonergic receptor binding in rhombic lip-derived regions of the medulla oblongata in the sudden infant death syndrome. J Neuropathol Exp Neurol 59:377-384

26. Paterson DS, Trachtenberg FL, Thompson EG, Belliveau RA, Beggs AH, Darnall R, Chadwick AE, Krous HF, Kinney HC 2006 Multiple serotonergic brainstem abnormalities in sudden infant death syndrome. JAMA 296:2124-2132

27. Kinney HC, Belliveau RA, Trachtenberg FL, Rava LA, Paterson DS 2007 The development of the medullary serotonergic system in early human life. Auton Neurosci 132:81-102

28. Machaalani R, Say M, Waters KA 2009 Serotoninergic receptor 1A in the sudden infant death syndrome brainstem medulla and associations with clinical risk factors. Acta Neuropathol 117:257-265

29. Ozawa Y, Okado N 2002 Alteration of serotonergic receptors in the brain stems of human patients with respiratory disorders. Neuropediatrics 33:142-149

30. Ozawa Y, Takashima S 2002 Developmental neurotransmitter pathology in the brainstem of sudden infant death syndrome: a review and sleep position. Forensic Sci Int 130:S53-S59

31. den Dunnen JT, Antonarakis SE 2000 Mutation nomenclature extensions and suggestions to describe complex mutations: a discussion. Hum Mutat 15:7-12

32. Wigginton JE, Cutler DJ, Abecasis GR 2005 A note on exact tests of HardyWeinberg equilibrium. Am J Hum Genet 76:887-893

33. Schoendorf KC, Hogue CJ, Kleinman JC, Rowley D 1992 Mortality among infants of black as compared with white college-educated parents. N Engl J Med 326:15221526

34. Price AL, Patterson N, Hancks DC, Myers S, Reich D, Cheung VG, Spielman RS 2008 Effects of cis and trans genetic ancestry on gene expression in African Americans. PLoS Genet 4:e1000294

35. Willinger M, James LS, Catz C 1991 Defining the sudden infant death syndrome (SIDS): deliberations of an expert panel convened by the National Institute of Child Health and Human Development. Pediatr Pathol 11:677-684

36. Krueger KC, Deneris ES 2008 Serotonergic transcription of human FEV reveals direct GATA factor interactions and fate of Pet-1-deficient serotonin neuron precursors. J Neurosci 28:12748-12758 